\title{
Etude au niveau des chloroplastes de modalités d'expres- sion de la résistance des Triticinées au piétin-verse
}

\author{
Marie-Christine SOULIE, Thérèse GUILLOT-SALOMON, Gérard DOUSSINAULT $\left(^{*}\right)$ \& Régis CAL- \\ VAYRAC $\left(^{* *}\right)$ \\ avec la collaboration technique de Catherine CANTREL $\left({ }^{*}\right)$ \\ Laboratoire de Biologie végétale IV, Université Pierre et Marie Curie, 12, rue Cuvier, F 75005 Paris \\ (*) I.N.R.A., Station d'Amélioration des Plantes, Centre de Recherches de Rennes, B.P. 29, F 35650 Le Rheu \\ (**) Laboratoire des Membranes biologiques, Tour 54-53, $3^{e}$ étage, Université Paris 7, 2, place Jussieu, $F$ \\ 75005 Paris
}

L'ultrastructure, la composition biochimique et l'activité physiologique des chloroplastes de diverses lignées de Triticinées sensibles et résistantes sont comparées après infection par Cercosporella herpotrichoïdes Fron, agent du piétin-verse. Quatre lignées de Triticinées sont choisies d'après leur niveau de résistance à la maladie : une lignée de blé tendre sensible « Moisson » (Triticum aestivum L.), 2 lignées résistantes «VPM » et « Roazon», issues respectivement du croisement (Aegilops ventricosa $\times$ Triticum persicum) $\times$ Marne $^{3}$ et de («VPM » $\times$ « Moisson»), et une lignée Aegilops ventricosa $n^{\circ} 11$ («Vent $\left.11 »\right)$ qui présente le plus haut niveau de résistance connu chez les Triticinées. Les chloroplastes sont extraits de la $1^{\text {ere }}$ feuille des plantules, à un stade précoce de l'infection, soit 3 à 4 semaines après l'inoculation.

L'analyse biométrique des données ultrastructurales ne révèle aucune différence dans l'organisation membranaire intraplastidiale chez les diverses lignées, après infection par $C$. herpotrichoides. Par contre, des différences notables sont décelées, simultanément, dans les teneurs en chlorophylles et en lipides polaires de ces organites. En effet, ces teneurs sont réduites chez la lignée «Moisson » et augmentées chez les lignées résistantes. Ces changements sont particulièrement spectaculaires pour « Roazon».

L'activité photosynthétique des chloroplastes, mesurée par le dégagement d'oxygène à la lumière, n'est affectée par la maladie que chez les lignées résistantes : le rendement photosynthétique baisse chez «VPM » alors qu'il augmente chez "Roazon » et « Vent 11 ».

Une légère résistance des chloroplastes au DCMU (3-(3,4 dichlorophényl 1,1-diméthylurée) a été détectée chez toutes les lignées. Cette résistance naturelle est modifiée au cours de la pathogenèse : elle est, soit inhibée chez « Roazon » et « Moisson», soit stimulée chez «VPM » et « Vent 11 ».

La diversité des réponses observées selon le niveau de résistance des plantes confirme l'existence d'une résistance polygénique au piétin-verse chez les Triticinées.

Mots clés additionnels : Ultrastructure, lipides, photosynthèse.

Ultrastructural, biochemical and physiological changes induced in chloroplasts from various susceptible or resistant lines of Triticineae, after infection with Cercosporella herpotrichoides Fron, the eyespot disease agent, were investigated. One line of susceptible bread wheat (Triticum aestivum L.) "Moisson ", two resistant lines "VPM" and "Roazon", issued from crossing betwcen (Aegilops ventricosa $\times$ Triticum persicum) $\times$ Marne $^{3}$ and ( VPM $\times$ " Moisson") respectively, and one line of Aegilops ventricosa Tausch («Vent $11 »)$ were studied. Chloroplasts were isolated from the first leaf of plants at an early stage of infection, 3 to 4 weeks after inoculation.

No significant difference in plastid ultrastructure could be detected for all lines after infection. On the other hand, changes were observed in the chlorophyll and polar lipid contents of these organelles. The amounts of these components decreased in the susceptible line « Moisson " but increased in the 3 resistant lines, mainly in the line "Roazon".

The photosynthetic activity of chloroplasts, measured by oxygen evolution in the light, was affected by the disease only in resistant lines. The photochemical activity was lower for "VPM" line and higher for " Roazon " and « Vent 11 ".

A slight resistance of plastids to DCMU could also be detected for all lines. This original property was either inhibited for « Roazon» and « Moisson », or stimulated for "VPM » and «Vent 11 ", after infection. These results suggest a polygenic resistance of Triticineae to cyespot disease.

Additional key words : Ultrastructure, lipids, photosynthesis. 


\section{INTRODUCTION}

Le piétin-verse est une maladie qui provoque d'importants dégâts aux cultures de blé dans les régions à climat tempéré humide (PONCHET, 1959). L'agent parasitaire responsable de la maladie est un champignon Cercosporella herpotrichoides Fron. Afin de lutter contre cette maladie, des lignées de blé à haut niveau de résistance au piétin-verse sont créées grâce à l'introduction des facteurs de résistance d'Aegilops ventricosa Tausch. chez le blé tendre (SIMONET, 1957 ; MaIA, 1967 ; Dosba \& DoussinaulT, 1978).

Les recherches actuelles s'orientent vers l'examen des mécanismes de résistance des plantes à cet agent pathogène. Ainsi, des études cytologiques ont précisé les modifications cellulaires qui interviennent au niveau des zones infectées d'hôtes sensibles (RASSEL, 1974 ; Defosse \& DeKEgEL, 1974 ; FehrmanN \& MENDGEN, 1975 ; Defosse, 1976), ou résistants (GUILlOT-SALOMON \& DoussinaUlT, 1981; KAMEL, 1981). Des variations de l'aspect ultrastructural du mycélium ont été signalées simultanément (GUILLOT-SALOMON \& DOUSSINAULT, 1981).

Il existe encore peu de recherches sur les effets du piétinverse sur l'activité physiologique des plantes. Seuls DAVY de VIRVILLE et al. (1981; 1982) montrent une forte inhibition de l'activité respiratoire des tissus infectés des lignées résistantes, alors qu'aucune modification de cette activité n'est signalée dans le même temps chez les lignées sensibles.

Des différences entre les lignées sensibles et résistantes sont observées, en l'absence d'infection, au niveau même du génome chloroplastique (VEDEL et al., 1978), ainsi que dans la composition polypeptidique des thylacoïdes plastidiaux (REMY et al., 1982).

Afin de détecter l'existence de modalités particulières d'expression des gènes de résistance des blés au piétinverse, nous recherchons les effets de la maladie, à la fois sur l'ultrastructure, la composition biochimique et l'activité physiologique des chloroplastes chez diverses lignées de Triticinées différant par leur niveau de résistance à la maladie.

\section{MATÉRIEL ET MÉTHODES}

\section{A. Matériel biologique : culture et infection}

Quatre lignées de Triticinées ont été choisies d'après leur degré de sensibilité ou de résistance au piétin-verse. La variété de blé tendre "Moisson», (Triticum aestivum L., ssp. vulgare), très sensible, obtenue à partir du croisement "Cappelle » $\times$ (Hybride 80-3 $\times$ «Etoile de Choisy »), a été comparée à 3 lignées résistantes : la $1^{\text {re }}, A$. ventricosa $\mathrm{n}^{\circ} 11$ ("Vent $11 »)$, est une lignée d'une espèce sauvage, à niveau de résistance particulièrement élevé ; la $2^{c}$ est le géniteur "VPM», blé tendre, issu du croisement $(A$. ventricosa $\times$ Triticum persicum) $\times$ Marne $^{3}$, dont une part importante de la résistance de l'Aegilops a été transférée dans son génotype ; la $3^{\mathrm{e}}$ est la variété "Roazon " sélectionnée dans la descendance du croisement ( VPM « $X$ « Moisson»). Cette dernière montre un niveau de résistance élevé tout en présentant des caractéristiques agronomiques favorables (rendement, résistance au froid, à la verse et à certains autres parasites).

Les caryopses, mis à germer en boîte de Petri à $19^{\circ} \mathrm{C}$, sont placés, dès l'apparition du coléoptile et des 3 racines séminales, dans des terrines contenant un mélange de terre et de sable. L'inoculation des plantules a lieu $3 \mathrm{j}$ après le semis selon la technique de MACER (1966), à l'aide d'une souche de $C$. herpotrichoïdes agressive et possédant une virulence de type blé (SCOTT et al., 1976). Cinq jours après l'inoculation, les plantules sont placées en chambre de vernalisation maintenue à $8^{\circ} \pm 1^{\circ} \mathrm{C}$ et éclairées avec une photopériode de $8 \mathrm{~h} / \mathrm{j}$ (tube Philips TLM 65W/55RS, fournissant une énergie de $10 \mathrm{~W} \cdot \mathrm{m}^{-2}$ ). Les plantules sont récoltées après 25 ou $32 \mathrm{j}$ de développement, selon les cas.

\section{B. Techniques cytologiques}

Des échantillons du limbe foliaire de $1 \mathrm{~mm}$ de côté sont prélevés à $2 \mathrm{~cm}$ de la pointe de la $1^{\mathrm{re}}$ feuille de plantules témoins et infectées âgées de $25 \mathrm{j}$. Après fixation pendant $90 \mathrm{mn}$ par une solution de glutaraldéhyde à $2,5 \mathrm{p}$. 100 dans du tampon cacodylate de $\mathrm{Na} 0,14 \mathrm{M}(\mathrm{pH} 7,4)$ et rinçage dans ce même tampon additionné de saccharose $0,4 \mathrm{M}$, on procède à une postfixation pendant $2 \mathrm{~h}$ à $4{ }^{\circ} \mathrm{C}$ par du tétroxyde d'osmium à 2 p. 100 dans ce même tampon. L'excès de tétroxyde d'osmium est éliminé par rinçage et les échantillons, déshydratés dans des bains d'alcool de degré croissant, puis d'oxyde de propylène, sont imprégnés par un mélange Durcupan ACM (Fluka). Les coupes, effectuées à l'aide d'un ultramicrotome LKB, sont contrastées à l'acétate d'uranyle-citrate de plomb (REYNOLDS, 1963).

\section{Analyse biométrique}

Afin d'apprécier quantitativement le développement des systèmes membranaires intraplastidiaux, des mesures sont effectuées à partir de 15 micrographies de sections longitudinales de chloroplastes obtenues selon leur plus grand axe. L'évaluation des longueurs des sections de thylacoïdes accolés et non accolés est réalisée à l'aide d'un curvimètre gradué en $\mathrm{mm}$. Les longueurs réelles des membranes sont obtenues en multipliant par 2 les valeurs mesurées, puis rapportées au $\mu \mathrm{m}^{2}$ de surface de section de plaste. Cette surface est déterminée par pesée selon la technique de PRIOUL \& BOURDU (1968). On définit ainsi l'encombrement membranaire qui désigne la surface totale de membranes $(\mathrm{Em}=$ longueur des membranes accolées + longueur des membranes non accolées), lâ longueur des partitions granaires ( $L p)$ et le pourcentage d'accolement (p. 100 accolement $=\frac{\mathrm{Lp} \times 2 \times 100}{\mathrm{Em}}$ ) (GUILLOT-SALOMON et al., 1978).

\section{Isolement des chloroplastes}

Les chloroplastes sont isolés de la partie apicale et moyenne de la $1^{\text {re }}$ feuille des plantules âgées de 25 ou $32 \mathrm{j}$ selon les cas. Deux méthodes sont utilisées selon qu'il s'agit de réaliser des dosages biologiques (méthode A) ou d'étudier les capacités photosynthétiques des chloroplastes (méthode B).

- Méthode $\mathrm{A}$ : les chloroplastes sont extraits à basse température $\left(0\right.$ à $\left.4{ }^{\circ} \mathrm{C}\right)$, selon une technique décrite antérieurement (GUillot-SALOMON et al., 1978). Après broyage des feuilles dans un milieu assurant une bonne intégrité ultrastructurale: saccharose $0,4 \mathrm{M}$, sérum albumine de bœuf $1 \mathrm{~g} / \mathrm{l}$, cystéine $0,5 \mathrm{~g} / 1, \mathrm{PO}_{4} \mathrm{HNa}_{2} 0,1 \mathrm{M}$ ( $\mathrm{pH} 7,8)$, et filtration de l'homogénat sur une toile de nylon (diamètre de la maille $100 \mu \mathrm{m}$ ), les chloroplastes sont précipités par centrifugation $(800 \mathrm{~g}$ pendant $5 \mathrm{mn})$, puis purifiés par centrifugation sur barrières de densité de 
saccharose : $5 \mathrm{M}(10 \mathrm{ml}), 1 \mathrm{M}(20 \mathrm{ml}), 0,75 \mathrm{M}(10 \mathrm{ml})$ $(1000 \mathrm{~g}$ pendant $10 \mathrm{mn})$. Les chloroplastes intacts $(80 \mathrm{à}$ 90 p. 100) sont recueillis à la partie inférieure de la couche de saccharose $1 \mathrm{M}$.

- Méthode B : afin d'assurer une bonne préservation des activités photosynthétiques ' des chloroplastes, le broyage des feuilles est effectué comme précédemment à $0-4^{\circ} \mathrm{C}$ dans le milieu suivant: sorbitol $0,33 \mathrm{M}$, tricine $1 \mathrm{mM}, \quad \mathrm{MgCl}_{2} 2 \mathrm{mM}, \quad(\mathrm{pH} 7,5)$. Après filtration de l'homogénat, les chloroplastes sont précipités par centrifugation $(800 \mathrm{~g}$ pendant $5 \mathrm{mn})$.

\section{E. Analyse biochimique}

Les dosages des chlorophylles sont effectués selon MACKINNEY (1941), celui des protéines selon LOWRY et al. (1951). Les lipides, extraits selon la méthode de FOLCH et al. (1957), sont séparés par chromatographie bidimensionnelle sur couche mince de gel de silice "MERCK 》 dans les phases solvantes: chloroforme-méthanol-eau $(65$ 25-4 V/V), puis chloroforme-méthanol-acétone-acide acétique-eau (100-20-40-20-10 V/V) et révélés par une légère exposition à des vapeurs d'iode. Le dosage des phospholipides est effectué par la technique de DUCET \& MENCL (1957) après minéralisation par l'acide sulfurique. Les galactolipides et le sulfolipide sont dosés selon la méthode de ROUGHAN \& BATT (1968). L'analyse des acides gras des lipides polaires est réalisée par chromatographie en phase gazeuse à partir de leurs esters méthyliques obtenus selon la technique de METCALFE et al. (1966).

\section{F. Mesure de l'oxygène dégagé au cours de la photo- synthèse}

L'étude du dégagement d'oxygène par les chloroplastes est réalisée à l'aide d'une électrode de type CLARK dans une cuve en pyrex. La température est maintenue à $25^{\circ} \mathrm{C}$ et les échanges sont mesurés en lumière blanche (lampe à filament de tungstène, Philips BA 21S fournissant une énergie de $520 \mathrm{~W} \cdot \mathrm{m}^{-2}$ ) (CALVAYRAC, 1970).

\section{RESULTATS EXPÉRIMENTAUX}

\section{A. Ultrastructure des chloroplastes}

Les chloroplastes du mésophylle des plantules âgécs de $25 \mathrm{j}$, saines (fig. 1A, 2A) et infectées (fig. 1B, 1C, 2B, 2C), présentent, pour toutes les lignées, un aspect ultrastructural classique. Leur système lamellaire interne est bien déve- loppé ; les empilements granaires sont nombreux et le plus souvent formés de l'accolement de 4 à 8 thylacoïdes.

L'analyse biométrique des données ultrastructurales a confirmé et précisé ces dernières observations. En effet, le pourcentage d'accolement de ces organites, qui définit la quantité de membranes de type granaire, est compris entre 50 et 60 p. 100 et leur encombrement membranaire, qui représente la quantité de membranes intraplastidiales, est proche de 20 à $30 \mu \mathrm{m}$ par $\mu \mathrm{m}^{2}$ de section de plaste (tabl. 1). Ces données sont caractéristiques des plantes dites de «soleil » (Guillot-SALOMON et al., 1978).

Aucune perturbation significative de l'organisation plastidiale n'est révélée simultanément lorsque les plantules sont infectées par $C$. herpotrichoïdes, et ceci quel que soit le niveau de résistance des lignées (tabl. 1). D'après ces résultats, la maladie ne semble pas modifier l'ultrastructure des chloroplastes des lignées sensibles ou résistantes.

\section{B. Composition biochimique des chloroplastes}

Les teneurs en chlorophylles et lipides polaires, composants majeurs des membranes des chloroplastes au même titre que les protéines, sont examinées chez les plantules âgées respectivement de 25 et de $32 \mathrm{j}$. Une étude préliminaire, ayant montré des modifications importantes du contenu pigmentaire et lipidique des feuilles chez les plantes infectées, alors que de faibles variations des tencurs en protéines sont signalées simultanément, les données biochimiques ont été rapportées à la teneur en protéines des chloroplastes.

\section{Teneur en chlorophylles}

D'après les données du tableau 2 , les chloroplastes de la lignée " Moisson " sont beaucoup plus riches en chlorophylles $a$ et $b$ que ceux des autres lignées, et notamment de la lignée "Vent 11 », la plus résistante des lignées examinées. En effet, chez cette dernière lignée, le taux en chlorophylles des chloroplastes de plantules témoins ne représente que 20 p. 100 de celui observé chez la lignée "Moisson". L'infection induit une légère diminution de ces teneurs chez la lignée "Moisson ", alors que la chlorophyllogenèse est, au contraire, stimulée (jusqu'à 30 p. 100 chez la lignée "Roazon») chez les lignées résistantes. Le rapport $\mathrm{Chl} \mathrm{a} / \mathrm{Chl} \mathrm{b}$ augmente simultanément chez les lignées «Vent $11 »$ et « Roazon».

\section{Teneur en lipides polaires}

L'analyse des lipides polaires totaux révèle, dans le cas des chloroplastes issus de plantules témoins, des taux beaucoup plus élevés chez la lignée «Moisson » que chez les

\section{TABLEAU 1}

Analyse biométrique de l'ultrastructure des chloroplastes de la $1^{r e}$ feuille de plantules âgées de 25 j, témoins (Te) et infectées (Inf) chez diverses lignées. Les données sont rapportées avec leur erreur standard sur chaque moyenne.

Biometric analysis of chloroplasts from the first leaf of control (Te) and infected (Inf) 25 day-old plants of various lines. Mean values with standard error.

\begin{tabular}{|c|c|c|c|c|c|c|c|c|}
\hline & \multicolumn{2}{|c|}{ Moisson } & \multicolumn{2}{|c|}{ Roazon } & \multicolumn{2}{|c|}{ VPM } & \multicolumn{2}{|c|}{ Vent 11} \\
\hline & $\mathrm{Te}$ & Inf & $\mathrm{Te}$ & Inf & $\mathrm{Te}$ & Inf & $\mathrm{Te}$ & Inf \\
\hline $\begin{array}{l}\text { Encombrement membranaire Em }\left(\mu \mathrm{m} / \mu \mathrm{m}^{2}\right) \\
\text { Longueur des partitions } \mathrm{Lp}\left(\mu \mathrm{m} / \mu \mathrm{m}^{2}\right)\end{array}$ & $\begin{aligned} 23 & \pm 6 \\
6 & \pm 2\end{aligned}$ & $\begin{array}{r}26 \pm 7 \\
7 \pm 2\end{array}$ & $\begin{aligned} 29 & \pm 5 \\
8 & \pm 2\end{aligned}$ & $\begin{array}{r}27 \pm 6 \\
7 \pm 2\end{array}$ & $\begin{aligned} 27 & \pm 7 \\
7 & \pm 2\end{aligned}$ & $\begin{array}{r}24 \pm 7 \\
7 \pm 2\end{array}$ & $\begin{aligned} 25 & \pm 9 \\
7 & \pm 3\end{aligned}$ & $\begin{array}{r}22 \pm 8 \\
6 \pm 2\end{array}$ \\
\hline$\%$ accolement $\frac{\mathrm{Lp} \times 2}{\mathrm{Em}} \times 100$ & $54 \pm 3$ & $56 \pm 5$ & $55 \pm 5$ & $49 \pm 3$ & $53 \pm 5$ & $56 \pm 4$ & $54 \pm 4$ & $56 \pm 4$ \\
\hline
\end{tabular}




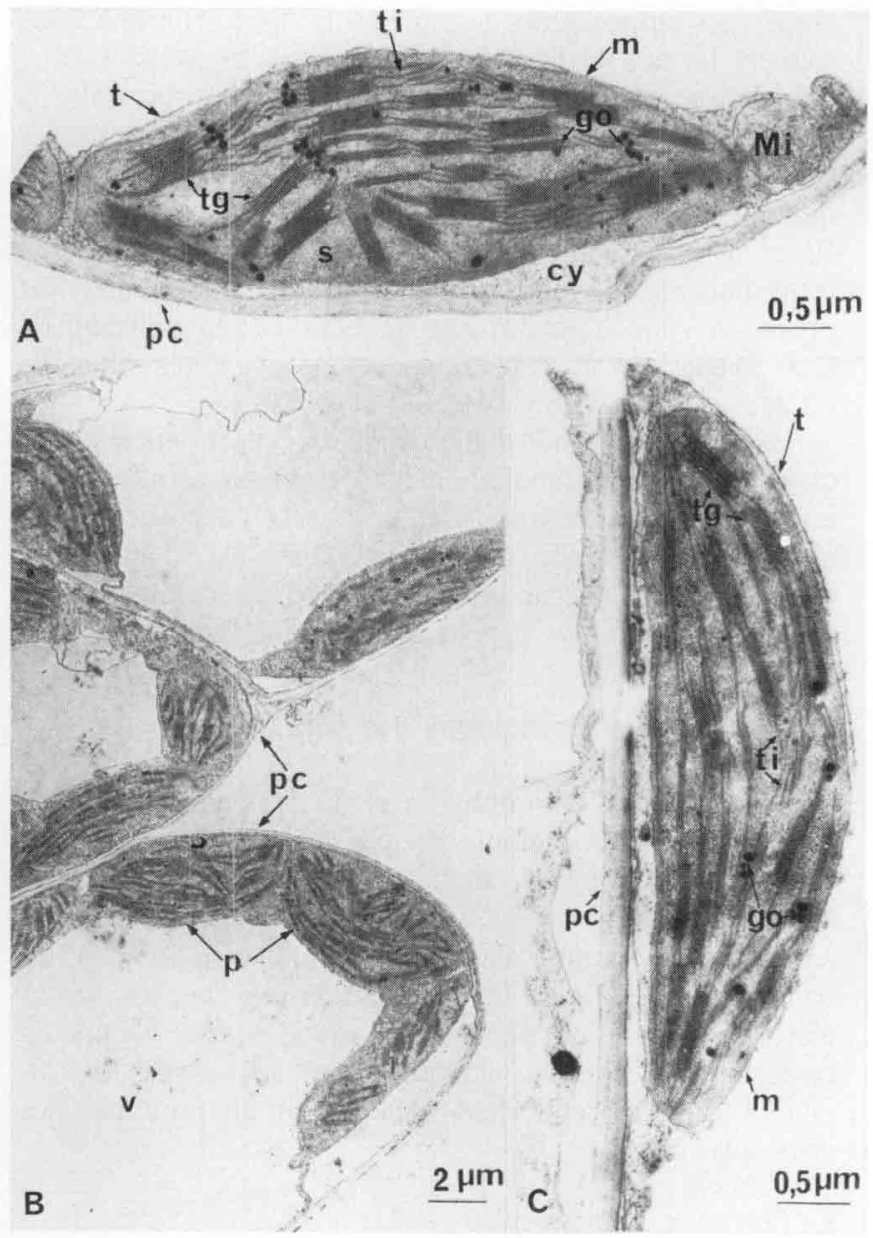

Figure 1

Ultrastructure des chloroplastes du parenchyme de la $1^{\text {re }}$ feuille de plantules témoin er infectée de la lignée sensible "Moisson".

A. Témoin.

B. et C. Infectée: B. Vue d'ensemble, C. Détail d'un plaste.

Chloroplast ultrastructure in the primary leaf parenchyma of control and infected susceptible line "Moisson".

A. Control.

$B$. and $C$. Infected : B. General view. C. Detail of a plastid.

cy: cytoplasme, go: globules osmiophiles, osmiophilic globules. $m$ : membrane plastidiale, plastid membrane. Mi: mitochondrie. $p$ : chloroplaste. PC: paroi pecto-cellulosique, pectocellulosic cell wall. $s$ : stroma. $t:$ tonoplaste. $1 \mathrm{~g}:$ thylacoilde granaire, grana thylakoid. $t i$ : thylacoide intergranaire, intergrana thylakoid. $v$ : vacuole

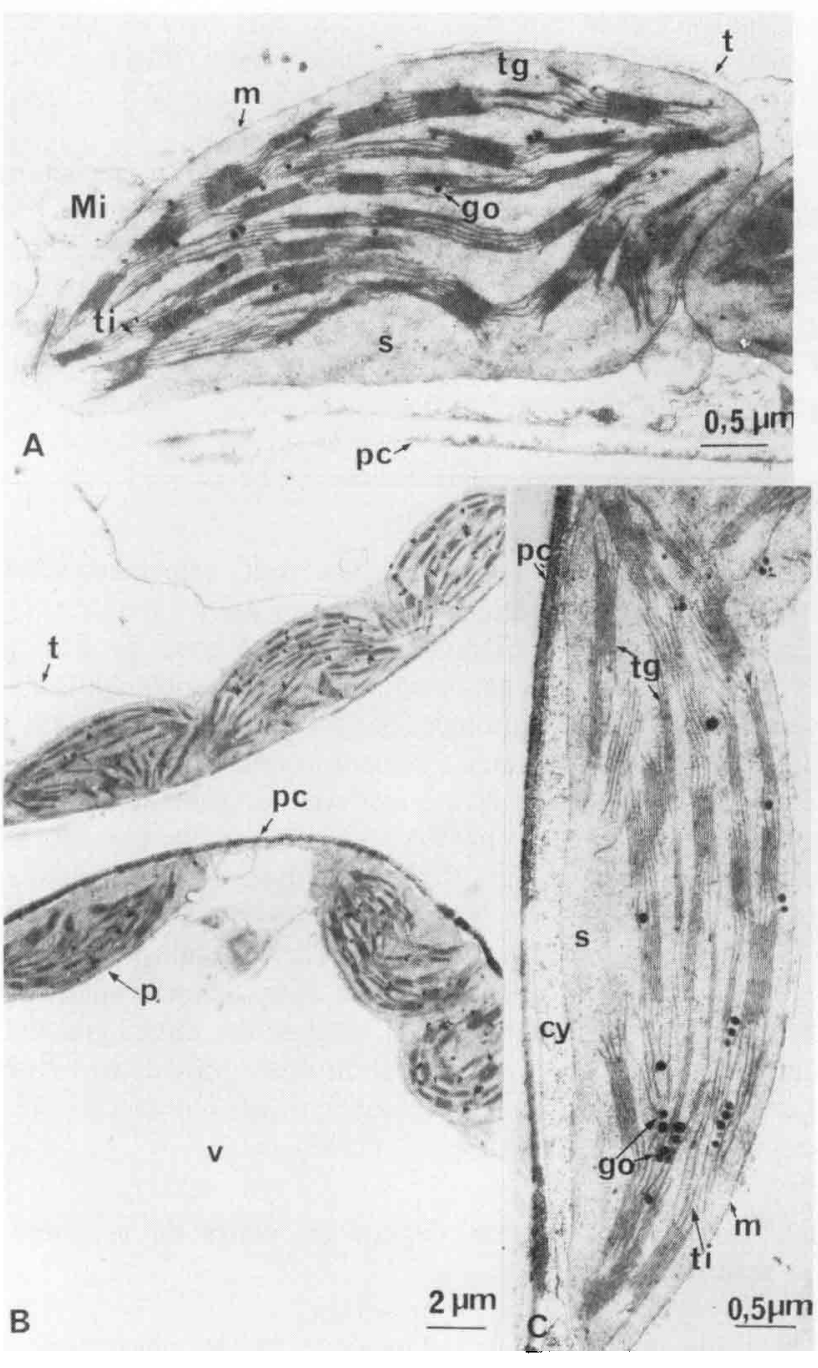

Figure 2

Ultrastructure des chloroplastes du parenchyme de la $1^{\text {re }}$ feuille de plantules témoin et infectée de la lignée résistante "VPM».

A. Témoin.

B. el C. Infectée: B. Vue d'ensemble, C. Détail d'un plaste.

Chloroplast ultrastructure in the primary leaf parenchyma of control and infected resistant line "VPM".

A. Control.

$B$. and $C$. Infected: B. General view, C. Detail of plastid.

TABLEAU 2

Teneurs en chlorophylles (en $\mu g$ par $m g$ de protéines) et activité de Hill mesurée par la quantité d'oxygène dégagée (en mol. O. $h^{-1} . m g$ Chl $l^{-1}$ ) des chloroplastes isolés de la $1^{\text {re }}$ feuille de plantules témoins (Te) et infectées (Inf) de diverses lignées. Les plastes sont extraits, soit de 300 plantules (teneurs en chlorophylles), soit de 25 plantules (activité de Hill) âgées de 32

Les données, rapportées avec leur erreur standard, sont les moyennes de 2 expériences.

Chlorophyll content (in $\mu g$ per $m g$ of proteins) and oxygen evolution (in mole. $\mathrm{O}_{2}$ per hour and per $\mathrm{mg}$ Chl) by chloroplasts from the first leaf of control (Te) and infected (Inf) 32 day-old plants of various lines. Plastids were isolated from 300 leaves (for chlorophyll content) or 25 leaves (for oxygen evolution). Mean values with their standard error from two experiments.

\begin{tabular}{|c|c|c|c|c|c|c|c|c|}
\hline & \multicolumn{2}{|c|}{ Moisson } & \multicolumn{2}{|c|}{ Roazon } & \multicolumn{2}{|c|}{ VPM } & \multicolumn{2}{|c|}{ Vent 11} \\
\hline & $\mathrm{Te}$ & $\operatorname{lnf}$ & $\mathrm{Te}$ & Inf & $\mathrm{Te}$ & Inf & $\mathrm{Te}$ & Inf \\
\hline $\begin{array}{l}\text { Chl } a \\
\text { Chl } b\end{array}$ & $\begin{array}{r}148 \pm 49 \\
47 \pm 15\end{array}$ & $\begin{array}{r}126 \pm 36 \\
40 \pm 10\end{array}$ & $\begin{array}{l}75 \pm 18 \\
24 \pm 7\end{array}$ & $\begin{aligned} 106 & \pm 23 \\
31 & \pm 8\end{aligned}$ & $\begin{array}{l}80 \pm 24 \\
25 \pm 8\end{array}$ & $\begin{array}{l}98 \pm 10 \\
31 \pm 5\end{array}$ & $\begin{array}{c}29 \pm 23 \\
9 \pm 7\end{array}$ & $\begin{array}{l}36 \pm 1 \\
10 \pm 3\end{array}$ \\
\hline$\frac{\text { Chl } a}{\text { Chl } b}$ & 3,2 & 3,1 & 3,1 & 3,4 & 3,2 & 3,2 & 3,2 & 3,6 \\
\hline Activité de Hill & $31 \pm 14$ & $29 \pm 14$ & $33 \pm 19$ & $40 \pm 17$ & $35 \pm 16$ & $24 \pm 15$ & $27 \pm 11$ & $43 \pm 14$ \\
\hline
\end{tabular}




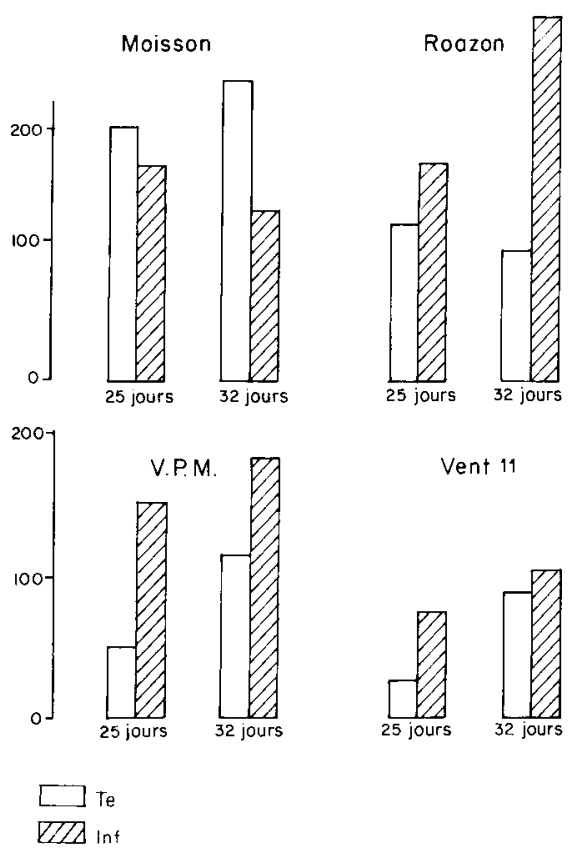

Figure 3

Teneur en lipides polaires totaux des chloroplastes isolés de la $I^{\text {re }}$ feuille de plantules témoins (Te) et infectées (Inf) de diverses lignées (en $\mu \mathrm{g}$ par $\mathrm{mg}$ de protéines).

Les plastes sont extraits de 300 plantules âgées de 25 et $32 \mathrm{j}$.

Total polar lipid content of chloroplasts from the first leaf of control (Te) and infected (Inf) 25 and 32 day-old plants of various lines (in $\mu \mathrm{g}$ per mg of proteins).

Plastids were isolated from 300 leaves.
3 autres lignécs (fig. 3). Ces taux augmentent entre le $25^{\circ} \mathrm{et}$ le $32^{\mathrm{c}} \mathrm{j}$ de croissance pour la plupart des lignécs, excepté pour la lignćc «Roazon» où une légère inhibition de ces synthèses intervient dans le même temps.

L'infection par le pathogène induit une baisse des teneurs en lipides polaires des chloroplastes de la lignée «Moisson ». Par contre, la synthèse de ces composés est stimulée chez les lignées résistantes. Ce phénomène est particulièrement net chez la lignée «Roazon » après 32 j (fig. 3 ).

L'examen des différents types de lipides polaires des chloroplastes confirme ces dernières observations (fig. 4). On remarque, en effet, que la lignée «Moisson" est beaucoup plus riche en chacun des galactolipides (mono- et digalactosyldiacylglycérol), de même qu'en chacun des phospholipides (phosphatidylglycérol notamment), que les autres lignées. Comme précédemment, on note une augmentation particulièrement spectaculaire des galactolipides chez la lignée "Roazon" ( $\times 3$ par rapport aux organites témoins) après $32 \mathrm{j}$ de croissance. Cette augmentation est moins sensible chez les lignées «VPM》 et «Vent 11 » après $32 \mathrm{j}$ de croissance.

L'analyse de la composition en acides gras de chaque catégorie de lipides n'a pas permis de détecter de différences notables entre les chloroplastes des plantes saines et infectées (tabl. 3). D'une manière générale, on retrouve la composition en acides gras des chloroplastes de la plupart des plantes de «soleil »(GUillot-SALOMON et al., 1978).

\section{Capacités photosynthétiques des chloroplastes}

Dans un premier temps, nous avons étudié l'activité photosynthétique des chloroplastes isolés des plantules
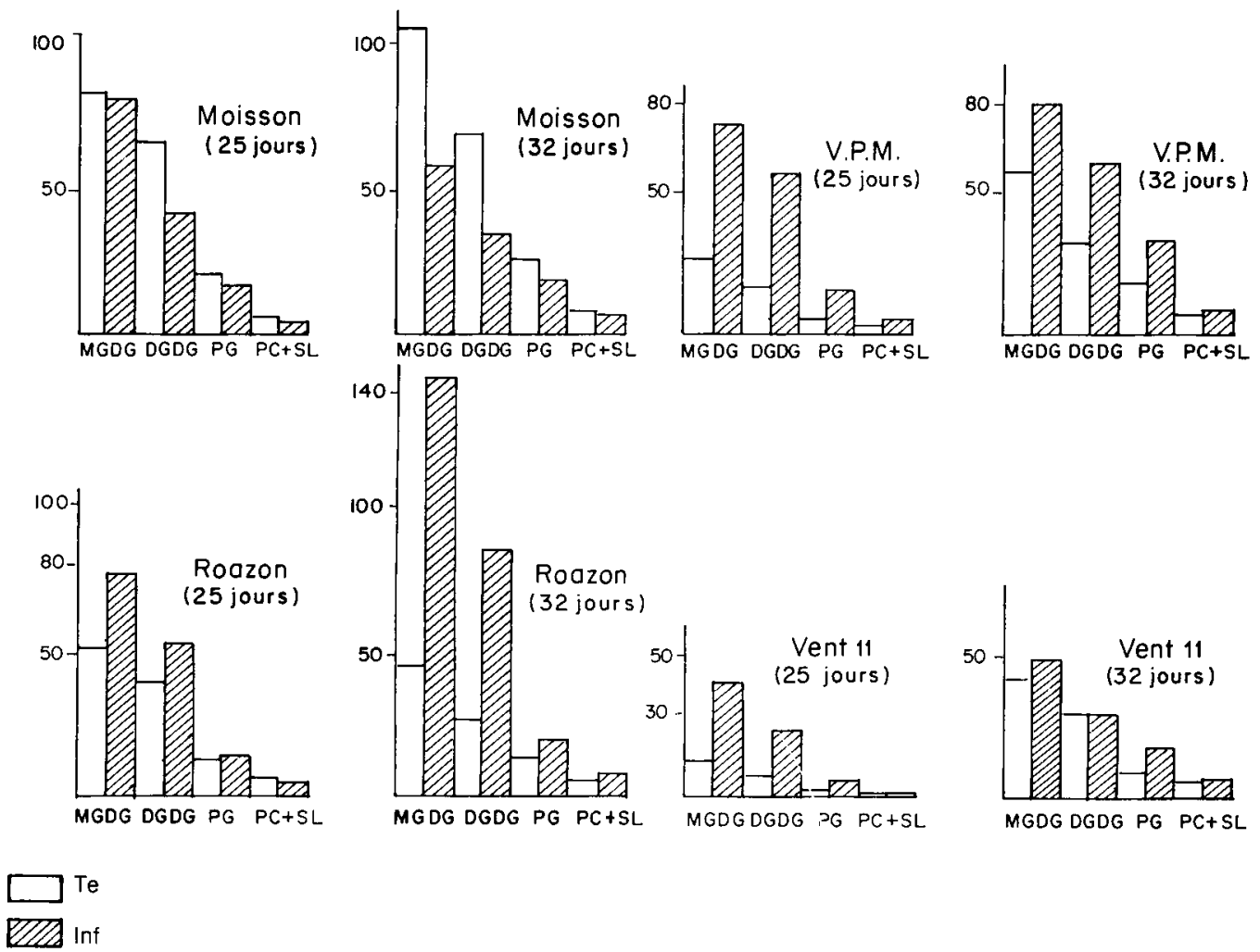

\section{Figure 4}

Composition en lipides polaires des chloroplastes isolés de la $I^{r e}$ feuille de plantules témoins (Te) et infectées (Inf) de diverses lignées (en $\mu g$ par $\mathrm{mg}$ de protéines).

Les plastes sont extraits de 300 plantules âgées de 25 ou de $32 j$.
Polar lipid composition of chloroplasts from the first leaf of control (Te) and infected (Inf) 25 and 32 day-old plants of various lines (in $\mu \mathrm{g}$ per $\mathrm{mg}$ of proteins).

Plastids were isolated from 300 leaves. 
TABLEAU 3

Composition en acides gras des lipides polaires des chloroplastes isolés de la $1^{\text {re }}$ feuille de plantules témoins et infectées des lignées sensible "Moisson "et résistante "Roazon" (en p. 100 des acides gras totaux de chaque lipide). Les plastes sont extraits de 300 plantules âgées de $32 j$. Fatty acid composition of plastid polar lipids from the first leaf of susceptible line "Moisson " and of resistant line "Roazon " (in \% of fatty acids). Plastids were isolated from 300 leaves of 32 day-old plants

MGDG et DGDG : mono- et digalactosyldiacylglycérol, $P C$ : phosphatidylcholine, $P G$ : phosphatidylglycérol, $S L:$ sulfoquinovosyldiacylglycérol, C 16 : acide palmitique, C 16:1 trans: acide trans-3 hexadécénoïque, C 18 acide stéarique, C 18:1: acide oléique, C 18:2: acide linoléique, C $18: 3$ : acide linolénique.

\begin{tabular}{|c|c|c|c|c|c|c|c|c|c|c|}
\hline \multirow[t]{2}{*}{ Acides gras } & \multicolumn{5}{|c|}{ Témoins } & \multicolumn{5}{|c|}{ Infectées } \\
\hline & MGDG & DGDG & $\mathrm{PC}+\mathrm{SL}$ & PG & $\begin{array}{l}\text { Lipides } \\
\text { totaux }\end{array}$ & MGDG & DGDG & $\mathrm{PC}+\mathrm{SL}$ & PG & $\begin{array}{l}\text { Lipides } \\
\text { totaux }\end{array}$ \\
\hline \multicolumn{11}{|c|}{ Lignée " Moisson " } \\
\hline C 16 & $t$ & 4 & 30 & 17 & 5 & $t$ & 4 & 24 & 17 & 9 \\
\hline C $16: 1$ & $t$ & $\mathrm{t}$ & - & 34 & 2 & $\mathrm{t}$ & $t$ & - & 39 & 3 \\
\hline C 18 & - & - & - & - & $\overline{1}$ & - & - & - & - & 3 \\
\hline C $18: 1$ & - & - & 一 & - & - & - & - & - & - & - \\
\hline C $18: 2$ & 2 & - & 4 & - & 2 & 3 & - & 3 & 3 & 2 \\
\hline C $18: 3$ & 98 & 96 & 66 & 49 & 90 & 97 & 96 & 74 & 41 & 83 \\
\hline \multicolumn{11}{|c|}{ Lignée "Roazon" } \\
\hline C 16 & $\mathrm{t}$ & 4 & 29 & 19 & 7 & $\mathrm{t}$ & 4 & 27 & 19 & 10 \\
\hline C $16: 1$ & $t$ & 2 & $\mathrm{t}$ & 37 & 4 & $\mathrm{t}$ & 1 & $\mathrm{t}$ & 38 & 4 \\
\hline C 18 & - & $\mathrm{t}$ & 1 & 1 & 3 & - & - & 1 & 2 & - \\
\hline C 18:1 & - & $\mathrm{t}$ & 1 & 2 & - & - & - & - & $\mathrm{t}$ & - \\
\hline C $18: 2$ & 3 & 1 & 5 & 5 & 2 & 2 & $\mathrm{t}$ & 4 & 4 & 3 \\
\hline C $18: 3$ & 97 & 93 & 64 & 36 & 84 & 98 & 95 & 68 & 37 & 83 \\
\hline
\end{tabular}

$\mathrm{t} \leqslant \%$.
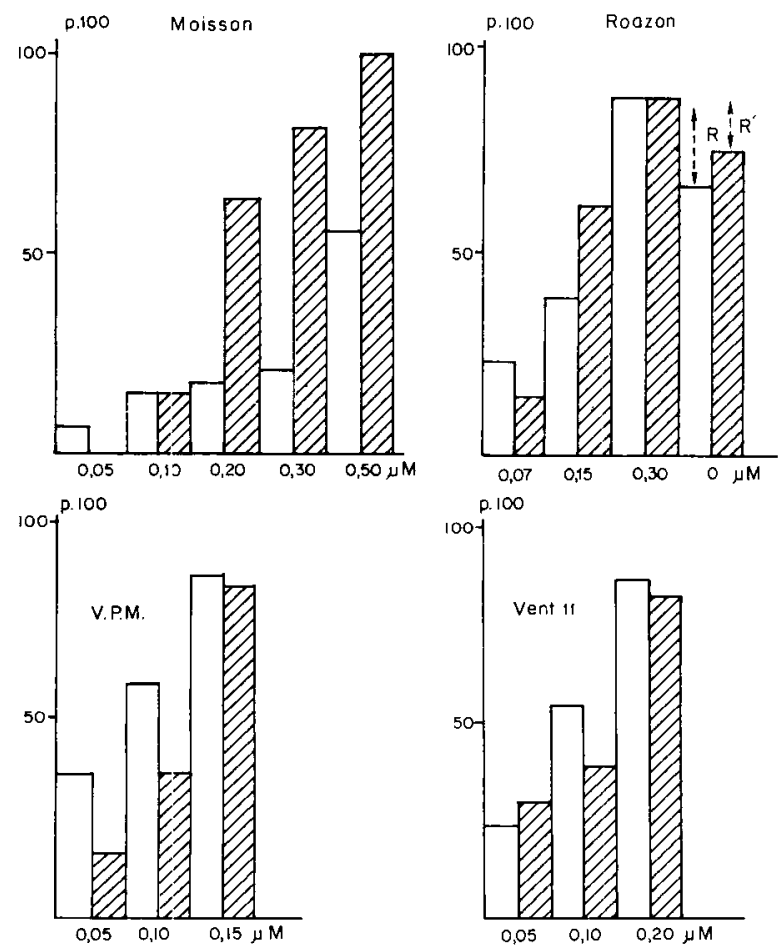

$\square$ Te

EZI inf

Figure 5

Pourcentage d'inhibition de l'activité photosynthétique des chloroplastes isolés de la $1^{\text {re }}$ feuille de plantules témoins (Te) et infectées (Inf) de diverses lignées en présence de différentes concentrations de DCMU $(\mu M)$.

$R$ et $R^{\prime}$ représentent le phénomène de "récupération".

Les chloroplastes sont extraits de 25 plantules âgées de $32 j$.

Percentage of photosynthesis inhibition after treatment of chloroplasts from the firsi leaf of control (Te) and infected (Inf) 32 day-old plants of various lines with different concentrations of DCMU ( $\mu M)$.

$R$ and $R^{\prime}$ : " recuperation " process.

Plastids were isolaied from 25 leaves. âgées de $32 \mathrm{j}$ en mesurant le dégagement d'oxygène de ces organites à la lumière et en présence de ferricyanure. Dans un second temps, nous avons examiné l'action à la fois du 3-(3,4-dichlorophényl)-1,1-diméthylurée (DCMU, inhibiteur spécifique du transfert des électrons entre les photosystèmes II et I, et du cyanure (CNK) qui agit sur les catalases ainsi que sur le transport des électrons entre les 2 photosystèmes.

\section{Mesure du dégagement d'oxygène des chloroplastes}

Dans des conditions de lumière blanche saturante (cf. II), l'addition d'un accepteur d'électrons tel que le ferricyanure entraîne un dégagement d'oxygène intense, comparable pour les 4 lignées examinées (tabl. 2). Cette réaction de Hill sc maintient au cours du temps.

Après infection des plantules par le champignon, l'activité photosynthétique des chloroplastes reste significativement inchangée chez la lignée « Moisson », alors qu'elle parait quelque peu modifiée chez les autres lignées. On constate ainsi une tendance à une diminution des taux d'oxygène dégagé à la lumière par les chloroplastes de la lignée «VPM » et une élévation, au contraire, de ces taux par les chloroplastes des lignées " Roazon " et "Vent 11 » lorsque les plantules sont parasitées. L'infection par C. herpotrichoïdes pourrait donc stimuler le rendement photosynthétique des chloroplastes chez ces 2 dernières lignées.

\section{Action du DCMU}

L'addition de faibles concentrations de DCMU $(0,05$ à $0,07 \mu \mathrm{M})$ ne bloque ni entièrement, ni de manière irréversible, le dégagement d'oxygène des chloroplastes extraits des feuilles des plantules saines des diverses lignées étudiées (fig. 5). Cette résistance au DCMU, nettement plus élevéc chez la lignée sensible «Moisson» (jusqu'à $0,3 \mu \mathrm{M}$ de DCMU) que chez les autres lignées (environ 0,05 à $0,15 \mu \mathrm{M}$ de DCMU selon les cas), est modifiée après infection par le 
piétin-verse. Elle est, soit levée, c'est le cas des lignées "Moisson " et " Roazon" pour des concentrations voisines de 0,2 à $0,15 \mu \mathrm{M}$ de DCMU, soit accrue, c'est le cas des lignées «VPM» et «Vent 11 », et ceci pour des concentrations voisines de $0,10 \mu \mathrm{M}$ de DCMU.

Une remarque intéressante concerne les chloroplastes de la lignée «Roazon». Chez ces organites, en effet, qu'ils soient issus de plantes infectées ou non, après l'inhibition partielle de la photosynthèse par addition de DCMU, suivie d'une brève période d'obscurité ( 3 à $4 \mathrm{mn}$ ), on constate, après une nouvelle illumination en lumière blanche saturante, une récupération partielle de la réaction de Hill (fig. 5). Ces résultats sont à rapprocher de ceux mis en évidence chez une lignée mutante d'Euglena gracilis $Z(Z R)$ qui possède des chloroplastes photosynthétiquement actifs et résistants à cet inhibiteur (CALVAYRAC et al., 1979a et $b$ ). Toutefois, seulement 22 p. 100 de l'activité photosynthétique est récupérée par les chloroplastes de la lignée «Roazon » alors que, chez l'euglène, la récupération de la photosynthèse s'élève à $70 \mathrm{p} .100$ de la photosynthèse initiale.

\section{Action du cyanure}

Comme l'ont montré Bomsel \& CALVAYRAC (1978) chez la lignée mutante d'euglène $(\mathrm{ZR})$ résistante au DCMU, l'addition de cyanure $(6,6 \mathrm{mM})$ au milieu de suspension de chloroplastes provoque l'inhibition totale et irréversible de la réaction de Hill chez toutes les lignées étudiées, qu'il y ait infection ou non par le piétin-verse. Les traces polarographiques ne montrent alors qu'une intense stimulation de la photoconsommation d'oxygène.

\section{DISCUSSION ET CONCLUSION}

L'ultrastructure des chloroplastes des tissus foliaires est identique pour toutes les lignées de Triticinées examinées. Celle-ci, de plus, ne semble pas perturbée par l'infection par $C$. herpotrichoïdes au stade plantule auquel nous avons effectué les observations. Par contre, des différences sont décelées entre lignées sensibles et résistantes au niveau de la composition biochimique et de l'activité physiologique de ces organites.

Les chloroplastes de la lignée "Moisson", la plus sensible des lignées étudiées, sont beaucoup plus riches en chlorophylles et en lipides polaires (phospholipides et galactolipides) que les autres lignées. Une forte réduction de la synthèse de ces composés est induite au cours de la pathogenèse. Chez les lignées résistantes, au contraire chez la lignée "Vent 11 » en particulier, la plus résistante et la plus pauvre en chacun des constituants examinés l'infection provoque une intense stimulation des synthèses pigmentaires et lipidiques. Cette stimulation est spectaculaire chez la lignée « Roazon».

D'une manière générale, la maladie induit donc, soit une inhibition de la synthèse des chlorophylles et des lipides polaires, composants spécifiques des thylacoïdes plastidiaux chez les lignées sensibles, soit une stimulation de l'élaboration de ces mêmes composés chez les lignées résistantes.

Les résultats obtenus pour la lignée sensible "Moisson " sont à rapprocher de ceux de LöSEL (1978), dans le cas des feuilles de Poa pratensis L. infectées par la rouille (Puccinia poarum Niels.). Cet auteur montre, en effet, une baisse rapide des quantités de galactolipides et de phosphatidylglycérol $(\mathrm{PG})$ dans ces tissus. Les teneurs en ces composés diminuent également lors du développement des spores de $P$. poarum chez Tussilago (LÖSEL \& LEWIS, 1974) ou d'urédospores d'Uromyces phaseoli (Pers.) Wint. chez Phaseolus vulgaris L. (HOPPE \& HEITEFUSS, 1975). Toute atteinte parasitaire au niveau foliaire semble donc se traduire par une forte réduction des teneurs en lipides plastidiaux. Ces mêmes changements sont observés par HUBER \& NEWMAN (1975) au sein des cotylédons sénescents de soja. Ils pourraient signifier, dans le cas du piétinverse, la stimulation ou l'induction de processus de sénescence au niveau des chloroplastes chez les hôtes sensibles.

Chez les lignées résistantes, où les synthèses membranaires sont, au contraire, activées en présence du parasite, on doit envisager la mise en jeu d'autres mécanismes, et notamment l'existence de récepteurs cellulaires chez l'hôte résistant, comme cela a été suggéré précédemment (GUILLOT-SALOMON \& DoussinaUlT, 1981). Ces récepteurs seraient susceptibles de reconnaître le parasite et d'induire une réaction qui, non seulement s'oppose au développement du champignon, mais active la synthèse des composés membranaires plastidiaux.

La communauté de réactions à l'infection des lignées résistantes au niveau de la composition biochimique des chloroplastes, peut être expliquée par l'examen de la généalogie des plantules (fig. 6). En effet, les lignées «VPM » et « Roazon » ont un ancêtre maternel commun, à savoir $A$. ventricosa d'où sont issus les caractères de résistance des blés en général. De plus, d'après VEDEL et al. (1981), les diagrammes de restriction des ADN chloroplastiques des lignées «Roazon» et «VPM» sont analogues à ceux du parent femelle $A$. ventricosa. Le fonctionnement des chloroplastes semble ainsi déterminé par leur propre information génétique en interaction avec celle provenant du noyau. La similitude des réponses recueillies par les 3 lignées de Triticinées résistantes après infection par C. herpotrichoïdes implique donc la transmission de caractères de résistance au piétin-verse liés au cytoplasme maternel. Ces caractères s'exprimeraient au moment de l'infection par la stimulation des synthèses pigmentaires et lipidiques au sein des chloroplastes.

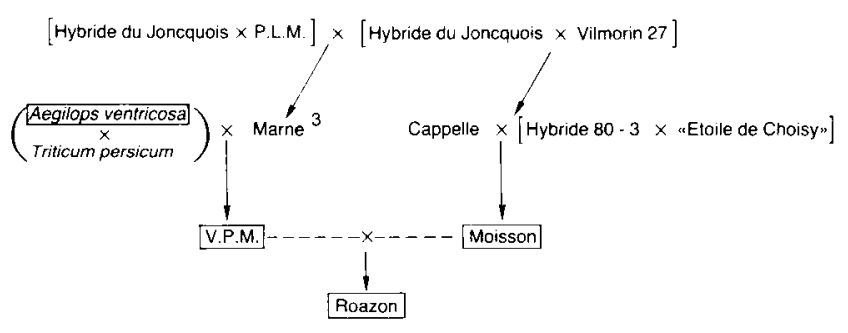

Figure 6

Généalogie des lignées de Triticinées étudiées.

Genealogy of the Triticineae lines studied.

Des différences entre lignées sensibles et résistantes sont également apparues lors de l'étude de l'activité photosynthétique de ces organites. En effet, cette activité ne semble pas modifiée par l'infection par $C$. herpotrichoïdes chez la lignée sensible "Moisson». Ces résultats vont à l'encontre des observations de PADHI et al. (1978) qui montrent chez le riz, après inoculation par Pyricularia oryzae Briosi et Cav., une considérable diminution de l'activité photosynthétique. Chez les lignées de Triticinées résistantes, au contraire, les capacités photosynthétiques 
des chloroplastes des plantes infectées sont perturbées. Deux effets opposés sont observés :

- soit le taux d'oxygène dégagé à la lumière diminue : c'est le cas de la lignée «VPM»;

- soit ce taux augmente: c'est le cas des lignées “ Roazon » et « Vent 11 ». La stimulation des synthèses des composés membranaires des chloroplastes, observée précédemment chez les lignées résistantes, ne s'accompagne donc pas nécessairement d'une exaltation des capacités photosynthétiques de ces organites.

La mise en évidence d'une différence entre les lignées "Roazon » et "Vent 11 ", d’une part, et la lignée "VPM", d'autre part, paraît difficile à interpréter d'après la généalogie des plantules. Toutefois, on peut avancer l'hypothèse que certains gènes, issus de la lignée «Vent 11 », s'exprimeraient chez la lignée "Roazon» et, au contraire, seraient réprimés chez la lignée «VPM».

L'action d'inhibiteurs photosynthétiques a révélé, de plus, une propriété liée génétiquement aux Triticinées étudiées, à savoir une certaine résistance naturelle au DCMU de leurs chloroplastes. Après infection, la résistance au DCMU est modifiée. Elle est stimulée chez les lignées «Vent 11 » et «VPM», ou partiellement inhibéc chez les lignées "Moisson" et "Roazon ". Le caractère de résistance au DCMU semble donc réprimé chez ces 2 dernières lignées. On pourrait en conclure que, en ce qui concerne les lignées résistantes, des gènes issus de la lignée «Vent 11 》 sont introduits chez la lignée «VPM», mais pas chez la lignée «Roazon». Quant à la levéc de résistance au DCMU, observée à la fois chez les lignées «Moisson» et « Roazon», elle pourrait provenir de la transmission de gènes de la lignée «Moisson » à la lignée « Roazon».

Les résultats obtenus démontrent donc l'implication de plusieurs gènes chloroplastiques dans la résistance des Triticinées au piétin-verse. Nos travaux confirment ainsi l'existence d'une résistance polygénique à cette maladie, déjà observée par JAHIER (1978).

L'examen des composants membranaires essentiels tels que les protéines, devrait permettre de confirmer cette dernière hypothèse. Il est, en effet, possible de détecter des différences au niveau du génome chloroplastique, comme l'ont démontré VEDEL et al. (1978) chez les lignées de Triticinées diploïdes telles qu'Aegilops squarrosa. De plus, REMY et al. (1982) ont précisé que ces différences se traduisent par la synthèse d'un polypeptide thylacoïdien de faible poids moléculaire, variable suivant la ploïdie de la lignée de blé. Le lien entre la présence de ce polypeptide et la résistance de Triticinées au piétin-verse reste toutefois à démontrer.

Reçu le 13 mars 1983 Accepté le 28 juillet 1983.

\section{RÉFÉRENCES BIBLIOGRAPHIQUES}

Bomsel J. L., Calvayrac R., 1978. Light-stimulated cyanide-inscnsitive oxygen uptake in Euglena gracilis $(Z)$, p. 299-306. In Plant mitochondria, G. Ducet, C. Lance eds., Elsevier North Holland Biomedical Press.

Calvayrac R., 1970. Relation entre les substrats, la respiration et la structure mitochondriale chez Euglena gracilis (Z). Arch. Mikrobiol., 73, 308-314.

Calvayrac R., Laval-Martin D., Dubertret G., Bomsel J. P., 1979a. Analysis and characterization of 3-(3,4 dichlorophenyl)-1,1dimethylurea (DCMU) resistant Euglena. II. Modifications affecting photosynthesis during adaptation to different doses of DCMU. Plant Physiol., 63, 866-872.

Calvayrac R., Ledoigt G., Laval-Martin D., 1979b. Analysis characterization of DCMU resistant Euglena gracilis. Planta, 145, 259-267.

Davy de Virville J., Lance C., Doussinault G., 1982. Réponse respiratoire au cours des premiers stades de l'infection chez diverses lignées de Triticinées sensibles ou résistantes à Cercosporella herpotrichoides. Cryptog., Mycol., 3, 319-332.

Davy de Virville J., Moreau F., Doussinault G., Lance C., 1981. Nature des interactions hôte-parasite lors de l'infection par Cercosporella herpotrichoïdes Fron de diverses lignées de Triticinées sensibles et résistantes. II. Etude de la respiration des tissus infectés. Agronomie, 1 (8), 695-700.

Defosse L., 1976. Recherches histochimiques sur la nature des réactions pariétales formées en réponse à la pénétration de Cercosporella herpotrichoïdes Fron dans le coléoptile du blé. Parasitica, 32, 147-157.

Defosse L., Dekegel D., 1974. Pénétration de Cercosporella herpotrichoides Fron $=$ Pseudocercosporella herpotrichoïdes (Fron) Deighton dans le coléoptile du froment (Triticum vulgare) observé en microscopic électronique. Ann. Phytopathol, 6 (4), 471-474.

Dosba F., Doussinault G., 1978. Création de lignées de blé présentant les caractéristiques agronomiques favorables d'Aegilops ventricosa. Ann. Amélior. Plant., 28, 27-44.

Ducet G., Mencl I., 1957. Séparation et dosage de faibles quantités de composés phosphatés «acido solubles». Ann. agron., 8 ( 2 bis), 17-31.

Fehrmann H., Mendgen K., 1975. Ultrastruktur von Wcrzenkoleoptilzellen nach Infektion mit Cercosporella herpotrichoides. Phytopathol. Z., 83, 267-380.
Folch J., Lees M., Sloane-Stanley G. H., 1957. A simple method for the isolation and purification of total lipids from animal tissues. $J$. Biol. Chem., 226, 497-509.

Guillot-Salomon T., Doussinault G., 1981. Nature des interactions hôte-parasite lors de l'infection par Cercosporella herpotrichoïdes Fron de diverses lignées de Triticinées sensibles et résistantes. I. Etude ultrastructurale des tissus au cours de la pathogenèse. Agronomie, 1 (4), 277-288.

Guillot-Salomon T., Tuquet C., de Lubac M., Hallais M. F., Signol M., 1978. Analyse comparative de l'ultrastructure et de la composition lipidique des chloroplastes de plantes d'ombre et de soleil. Cytobiol., 17, 442-452.

Hoppe H. H., Heitefuss R., 1975. Permeability and membrane lipid metabolism of Phaseolus vulgaris infected with Uromyces phaseoli. IV. Phospholipids and phospholipid fatty acids in healthy and rust infected bean leaves resistant and susceptible to Uromyces phaseoli. Physiol. Plant Pathol., 5, 263-490.

Huber D. J., Newman D. W., 1975. Relationships between lipid changes and plastid ultrastructural changes in senescing and regreening soybean cotyledons. J. exp. Bot., 27, 490-511.

Jahier J., 1978. Etude des relations hôtes-parasites, aux stades plantules et adultes chez les Triticinées, dans le cas de Cercosporella herpotrichoïdes Fron, agent du piétin-verse. Thèse Doct. Agronomie Rennes, $\mathrm{n}^{\circ} 78-1,87 \mathrm{p}$.

Kamel Y., 1981. Etude comparative du Cercosporella herpotrichoïdes Fron chez des hôtes respectivement sensible, résistant et très résistant. Thèse Doct. $3^{\mathrm{e}}$ cycle. Univ. P. M. Curie, Paris, 81 p.

Lösel D. M., 1978. Lipid metabolism of leaves of Poa pratensis during infection by Puccinia poarum. New Phytol., 80, 167-174.

Lösel D. M., Lewis D. H., 1974. Lipid metabolism in leaves of Tussilago farfara during infection by Puccinia poarum. New Phytol., 73, 1157.

Lowry O. H., Rosebrough N. J., Farr A. L., Randall R. J., 1951. Protein measurement with the Folin phenol reagent. J. Biol. Chem., 193, 265-275.

Macer R. C. F., 1966. Resistance to eyespot disease (Cercosporella herpotrichoides Fron) determined by a scedling test in some forms of Triticum, Aegilops, Secale, Hordeum. J. agric. Sci., 67, 389-396. Mackinney G., 1941. Absorption of light by chlorophyll solutions. J. Biol. Chem., 140, 315-322. 
Maia N., 1967. Obtention de blés tendres résistants au piétin-verse (Cercosporella herpotrichoïdes) par croisements interspécifiques. C.R. Acad. Agric. Fr., 53, 149-154.

Metcalfe L. D., Schmitz A. A., Pelka J. R., 1966. Rapid preparation of fatty acids csters from lipids for gas chromatographic analysis. Ann. Chem., 38, 514-515.

Padhi B., Chakrabarti N. K., Nayak S. K., 1978. Effect of blast infection on photosynthetic activity of rice seedling. Biol. Plant., 20, $418-420$

Ponchet J., 1959. La maladie du piétin-verse des céréales Cercosporella herpotrichoïdes Fron. Importance agronomique, biologie, épiphytologie. Ann. Epiphyties, 10, 45-98.

Prioul J. L., Bourdu R., 1968. Utilisation de l'analyse biométrique à l'étude de la dynamique des infrastructures chloroplastiques lors de la levée d'une carence en azote. J. Microscopie, 7, 419-439.

Rassel A., 1974. Observation en microscopie électronique de cellules mycéliennes de Cercosporella herpotrichoïdes Fron dans les cellules de gaines foliaires de froment. Ann. Phytopathol., 6, 25-34.

Rémy R., Ambard-Bretteville F., Vedel F., 1982. EcoRI analysis of chloroplast DNAs and polypeptide composition of thylakoids from wheat and related species. Plant. Sci. Lett., 25, 261-270.

Reynolds E. S., 1963. The use of lead citrate at high $\mathrm{pH}$ as an electron opaque stain in electron microscopy. J. Cell. Biol., 17, 208212.

Roughan P. G., Batt R. D., 1968. Quantitative analysis of sulfolipids (sulfoquinovosyldiglyceride and galactolipids, monogalactosyland digalactosyldiglycerides) in plant tissues. Anal. Biochem., 22, 74-88.

Scott P. R., Defosse L., Vandam J., Doussinault G., 1976. Infection of lines of Triticum. Secale. Aegilops and Hordeum by isolates of Cercosporella herpotrichoides. Trans. Br. mycol. Soc., 66, 205-210. Simonet M., 1957. Hybrides interspécifiques et intergénériques. Ann. Amélior. Plantes, 4, 395-411.

Vedel F., Quetier F., Cauderon Y., Dosba F., Doussinault G., 1981. Studies on maternal inheritance in polyploid wheats with cytoplasmic DNAs as genetic markers. Theor. appl. Genet., 59, 239-245. Vedel F., Quetier F., Dosba F., Doussinault G., 1978. Study of wheat phylogeny by EcoRI analysis of chloroplastic and mitochondrial DNAs. Plant Sci. Lett., 13, 97-102. 